\section{(6) OPEN ACCESS}

\title{
Cancer arising from the remnant mucosa of the ileoanal anastomosis leading to pouchectomy
}

\author{
Leon Gearoid Walsh, ${ }^{1,2}$ Bryan J Kenny, ${ }^{3}$ Mazen El Bassiouni, ${ }^{4}$ John Calvin Coffey ${ }^{1,2,5}$
}

${ }^{1}$ Department of Surgery, University Hospital Limerick, Limerick, Limerick, Ireland 2Department of Surgery, University of Limerick GEMS, Limerick, Limerick, Ireland ${ }^{3}$ Department of Radiology, University Hospital Limerick, Limerick, Limerick, Ireland ${ }^{4}$ Cancer Centre, University Hospital Limerick, Limerick, Limerick, Ireland

${ }^{5}$ Centre for Interventions in Infection, Inflammation and Immunity, Graduate Entry Medical School, University of Limerick, Limerick, Ireland

\section{Correspondence to}

Dr Leon Gearoid Walsh, leongwalsh@icloud.com

Accepted 3 June 2016

\section{SUMMARY}

Ileal pouch-related adenocarcinoma remains a rarity; thus, guidelines on treatment are currently lacking.

We present this case of a 54-year-old man who underwent restorative proctocolectomy with stapled ileal pouch-anal anastomosis formation for familial adenomatous polyposis during the 1980s. Despite undergoing annual surveillance endoscopy, the patient was noted to be anaemic and passing fresh blood per anus. Endoscopy and radiological investigation revealed the presence of a pouch-related adenocarcinoma. This was subsequently treated with short-course radiotherapy and pouch excision. The patient remains well until now and will follow six-monthly surveillance protocols with a transition to annual surveillance after 2 years.

\section{BACKGROUND}

Since ileal pouch cancer remains a rarity, treatment guidelines and protocols are lacking. ${ }^{1}$ This presents a difficulty in their management. Optimal outcomes in rectal cancer are achieved when combined treatment modalities (ie, chemotherapy and radiotherapy) are followed by mesenteric-based surgery. ${ }^{2-4}$ Examples of the latter include total mesorectal excision, total mesocolic excision and complete mesocolic excision. The technical demands of these approaches increase dramatically in the reoperative context where mesenteric planes (ie, mesofascial plane) may be difficult to identify amid adhesional complexes encountered.

In keeping with this, we present the case of a patient with a history of familial adenomatous polyposis (FAP) who, despite regular surveillance, developed a pouch adenocarcinoma. The patient underwent neoadjuvant short-course radiotherapy followed by surgery involving a strictly anatomicbased pouch excision.

\section{CASE PRESENTATION}

We report the case of a 54-year-old man with a background of restorative proctocolectomy and stapled ileal pouch anal anastomosis performed in 1987 for FAP. The pathology report from the index operation indicated multiple adenomatous polyps throughout the colon. There was no evidence of malignancy. Following this, the patient underwent yearly surveillance pouchoscopy and upper gastrointestinal endoscopy. Prior to referral, he was anaemic and passing fresh blood per anus. There was no history of FAP in this patient's family. This was a de novo adenomatous polyposis coli (APC) genetic mutation in this patient. Genetic mutation-specific testing yielded negative results in this man's offspring.

\section{INVESTIGATIONS}

Pouchoscopy demonstrated a suspicious mucosal lesion in the distal pouch and biopsies confirmed an adenocarcinoma. Histologically, the epithelial features were most in keeping with colorectal mucosa pointing to the possibility of either a retained rectal remnant or normal pouch-related epithelial metaplasia. Oesophagogastroduodenoscopy was normal. Staging CT demonstrated irregular soft-tissue attenuation within the ileal pouch consistent with pouchoscopy findings (figure 1). Abdominal CT identified extensive mesenteric lymphadenopathy along the distribution of the superior mesenteric artery (SMA). An incidental finding of a left adrenal gland lipid-rich adenoma was made and confirmed using an adrenal-based CT protocol. The liver, spleen, pancreas, lungs and contralateral adrenal gland were radiologically normal. MRI demonstrated signal abnormality of the posterior ileal pouch without visible extension through the bowel wall (figure 2). Two large lymph nodes were noted, each with a diameter of $1.5 \mathrm{~cm}$, in the pouch mesentery. Endoscopic ultrasound demonstrated a well-defined lobulated solid mass lesion arising from mucosa, extending posteriorly and penetrating into overlying mesenteric fat between the 6 and 8 o'clock positions. This pouch mass was located immediately above the upper anal sphincteric plate. Abnormally enlarged lymph nodes were also noted adjacent to the SMA where the latter exits the pancreas to enter the small intestinal mesentery. Final preoperative radiological staging was $\mathrm{T} 3 \mathrm{~N} 1 \mathrm{~b} \mathrm{Mx}$.

\section{TREATMENT}

The multidisciplinary consensus recommended short-course neoadjuvant radiotherapy in view of the possibility of lymphatic metastatic disease at an

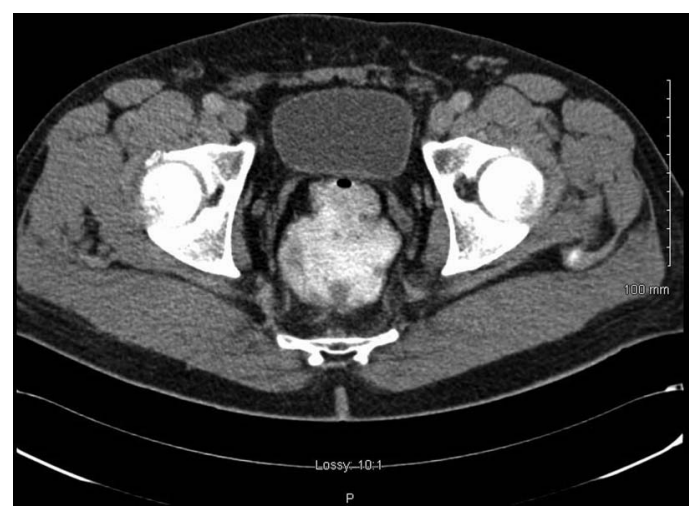

Figure 1 Intravenous and oral contrast computerised axial tomography demonstrating an ileal pouch. 


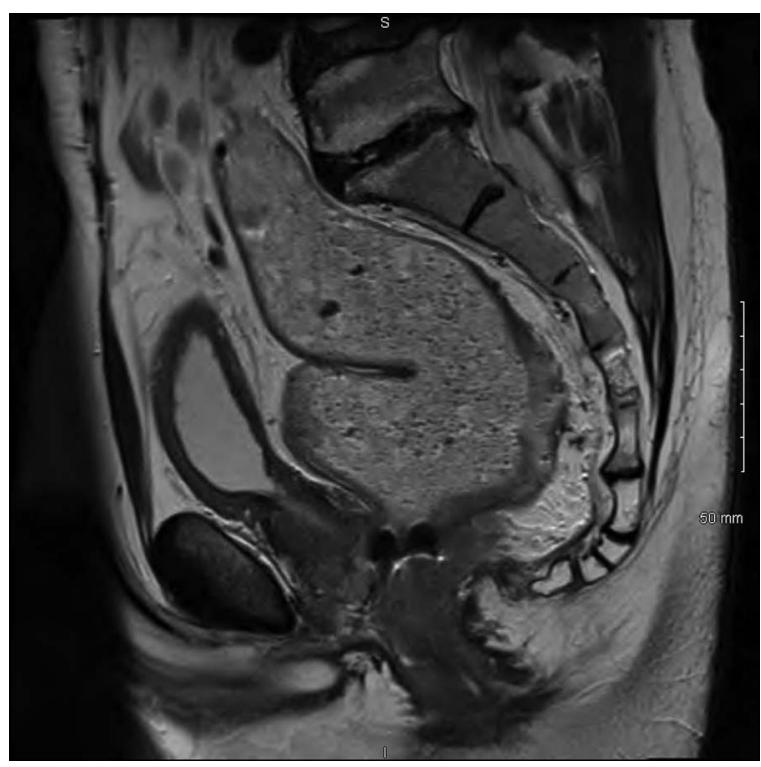

Figure 2 T2-weighted MRI in a sagittal view of ileal pouch in situ postproctocolectomy. There is an area of ill-defined signal intensity along the posterior aspect of the pouch without visible extension through the bowel wall.

extraregional location. A cumulative dose of 25 Gy externalbeam radiotherapy was delivered over 5 consecutive days. The patient proceeded to surgery after 1 week of treatment completion and was admitted for elective resection of the ileal pouch and anal canal, completion mesenterectomy and end ileostomy.

\section{Abdominal component}

Following general anaesthesia, two $4.8 \mathrm{Fr} \times 24 \mathrm{~cm}$ ureteric stents were placed under cystoscopic guidance. A midline laparotomy was performed through an old scar. Extensive, dense adhesions were noted and careful adhesiolysis was performed. In this manner, the small intestinal mesentery was detached from the retroperitoneum without breach, and retaining Toldt's fascia intact. The small intestine and associated mesentery was divided $20 \mathrm{~cm}$ proximal to the ileal pouch and $\sim 300 \mathrm{~cm}$ of small intestine remained proximal to this. Once the plane between the small intestinal mesentery and retroperitoneum was identified, it was followed to guide the pelvic dissection. The latter was carried out down to the pelvic floor circumferentially. Radiation-related changes meant that the mesentery was attached (but not fused) to the surrounding tissue. Using the above approach to mobilisation, the entire mesenteric package was maintained intact and fully detached to the pelvic floor level. Finally, individual lymph nodes were resected along the SMA up to the level where it emerged from the body of the pancreas. An end ileostomy was formed and matured in the right flank.

\section{Perineal component}

In the prone position, the buttocks were taped back and the anal canal closed using a purse-string suture. A church-window skin incision was made around the anal canal and deepened through the fat of the ischioanal space to the pelvic floor. The latter was fully exposed circumferentially. The anococcygeal raphe was divided in the posterior midline and the division extended anteriorly in a circumferential manner. As a result, the levator muscle was included in the resection, without going wide enough to endanger the pudendal nerve and vessel in
Alcock's canal. The ileal pouch was subsequently mobilised from the prostate, vas deferens and seminal vesicles. The entire specimen was exteriorised en bloc and inspected. The perineal wound was closed using 2.0 Vicryl sutures in layers and the skin wounds were apposed with 1.0 Ethilon nylon sutures in an uninterrupted fashion.

\section{OUTCOME AND FOLLOW-UP}

Postoperatively, pain was controlled using a morphine-based patient-controlled analgesia and regular intravenous paracetamol. Ureteric stents were removed in the postoperative period. Early postoperative recovery was uncomplicated and the patient discharged. At follow-up, the patient reported of perineal pain and CT investigation demonstrated a $4 \mathrm{~cm}$ pelvic collection. This was treated with intravenous antibiotics after which he was transitioned to oral therapy. The collection discharged through the perianal wound and symptoms resolved.

Postoperative histological examination confirmed a $5 \mathrm{~cm}$ tumour at the anastomotic site. Microscopy revealed moderate to poorly differentiated adenocarcinoma with infiltration through the bowel wall. Lymphovascular invasion was not seen. The circumferential, proximal and distal resection margins were clear of malignancy. Four of 20 resected lymph nodes (in the pouch mesentery) had metastatic adenocarcinoma. The second anastomotic site was free of tumour. Lymph nodes excised from the root of the SMA were free of metastatic disease. Final surgical staging was pT3 N2a Mx G2 adenocarcinoma of the ileal pouch. At the latest follow-up, his pelvic pain settled and all wounds were healed. The patient declined adjuvant chemotherapy. Future surveillance will be six monthly followed by a transition to annual after 2 years. As per centre protocol, serum carcinoembryonic antigen levels will be assessed going forward.

\section{DISCUSSION}

Although 22 cases of FAP-related ileal pouch adenocarcinoma have been reported, ${ }^{5}{ }^{6}$ this is the first reported case of a patient who developed an adenocarcinoma within an ileal pouch and who underwent neoadjuvant short-course radiotherapy, followed by pouchectomy. Of ileal pouch recurrences, the duration between index surgery and recurrence is between 3 and 33 years (median=10 years). Seven patients were diagnosed with stage II disease with a further six stage III reported. Additionally, Tajika et $a l^{6}$ reported 1 stage I and 1 stage IV patient with ileal pouch recurrence. In theory, patients undergoing ileal pouch-anal anastomosis (IPAA) for FAP should also undergo mucosectomy, thereby reducing the risk of further cancer development. ${ }^{1}$ However, some of the earliest pouches fashioned may not have had an associated mucosectomy. Even despite synchronous mucosectomy at the time of surgery, small areas of residual rectal mucosa may remain; thus, the risk of adenocarcinoma recurrence persists. ${ }^{5}$ This is notably the case in patients undergoing a double-stapled anastomosis and has been demonstrated histologically. ${ }^{15-7}$ Centralisation and standardisation of the technical approaches to index proctocolectomy and pouch formation could reduce the variation of amounts of residual rectal mucosa seen, thus inferring more favourable outcomes in this patient cohort. However, should development of adenocarcinoma in residual rectal mucosa occur, this may best be managed in accordance with principles of management of rectal cancer (ie, staging, neoadjuvant chemoradiotherapy and adjuvant chemotherapy).

Short-course preoperative pelvic radiotherapy involves delivery of $25 \mathrm{~Gy}$ in five daily treatments over 1 week. It uses megavoltage X-rays (15 MV) delivered in a four-field box conformal technique. This is followed by surgery 1 week later. The 
Swedish Rectal Cancer Trial and the Dutch Commisse Klinisch Vergelijkend Onderzoek (CKVO) 95-04 trial demonstrated improvements in local cancer control with short-course radiotherapy. ${ }^{8} 9$ This study also indicated that best results are achieved when the short course is combined with total mesorectal excision. ${ }^{9}$ Traditionally, long-course chemoradiotherapy delivers $\sim 50.4$ Gy of radiation in 28 fractions concurrently with chemotherapy followed by surgery within 6 weeks. ${ }^{10}$ However, radiation dosage and duration of treatment may vary between centres. Additionally, the optimal interval between completion of chemoradiation and surgery remains unconfirmed. ${ }^{11}$ The German Rectal Cancer Study used an interval of 6 weeks between completion of neoadjuvant treatment and surgery; consequently, most centres have incorporated this timing. This study demonstrated improved local cancer control, reduced rates of recurrence and increased sphincter preservation with combined neoadjuvant modalities. ${ }^{10}$

Short-course radiotherapy was used in the case presented here, given the radiological evidence of mesenteric lymphatic metastases near the origin of the SMA. In the case presented, early surgery was advocated given (1) the duration of longcourse treatment and (2) concerns of SMA nodal spread.

Debate persists regarding the relative benefits between shortcourse and long-course chemoradiotherapy regimens. Cross-trial comparisons between both failed to identify differential benefits. ${ }^{12}$ In a randomised trial comparing both, Ngan et al identified lower cumulative local recurrence rates in low rectal adenocarcinoma at 3 and 4 years in patients assigned to the long-course chemoradiotherapy regimen group. However, the difference was not statistically significant. ${ }^{13}$ The Trans-Tasman Radiation Oncology Group (TROG) study revealed no significant differences in toxicity and overall survival between both types of treatment. ${ }^{13}$ This corroborated the conclusions made in the Polish long-term trial comparing the radiotherapy and chemoradiotherapy cohorts. ${ }^{12}$

Our case illustrates the benefits of applying mesenteric principles in complex reoperative surgery. The abdominal component was conducted according to strict mesenteric principles. Accordingly, the mesofascial plane between the small intestinal mesentery and subjacent retroperitoneum was identified and used as an anatomic road map down into the pelvis. Although planar components were attached and oedematous, they were not fused, which meant that the mesenteric package was maintained entirely intact. By adhering to the mesofascial plane, serosal tears and full thickness enterotomy were entirely avoided and blood loss minimised. The mesofascial plane was followed proximally to the origin of the mesentery where the SMA exits from the pancreas. In this case, enlarged lymph nodes present in this location were also excised.

In summary, it is well established that colectomy with ileorectal anastomosis does not entirely remove the risk of metachronous cancer development in $\mathrm{FAP}^{2}$ A risk of cancer development remains following proctocolectomy and IPAA formation. Smith et $a l^{5}$ suggest that the risk of malignancy following surgery for FAP increases with time. ${ }^{2}$ Therefore, despite the adopted procedure, the risk of cancer recurrence following surgery for FAP remains such that intensive surveillance endoscopy should be adopted to identify pouch adenomas and tumours early in their natural history. Notwithstanding this, IPAA formation remains an appropriate treatment option for patients with FAP with a good postoperative quality of life and functional outcomes. ${ }^{1}$ Should a pouch adenocarcinoma arise, neoadjuvant short-course radiotherapy followed shortly after by strictly anatomic-based pouchectomy may be safely conducted.

\section{Learning points}

- The risk of metachronous cancer development in patients with familial adenomatous polyposis (FAP) is not eliminated following proctocolectomy and ileal pouch-anal anastomosis (IPAA) formation.

- Intensive surveillance endoscopy should be adopted in order to identify pouch-related adenomas and tumours early in their natural history.

- Should a pouch adenocarcinoma be identified, neoadjuvant short-course radiotherapy followed by pouchectomy along strict anatomic planes may be safely conducted.

- Restorative proctocolectomy with IPAA formation remains the surgical standard of treatment for patients of FAP.

Contributors LGW contributed as the first author to the paper and as a part of the surgical team caring for the particular patient. BJK conducted radiological studies as outlined in the paper. MEB delivered neoadjuvant radiotherapy as per the paper. JCC was the surgical consultant performing the surgery and leading in the care of this patient.

Competing interests None declared.

Patient consent Obtained.

Provenance and peer review Not commissioned; externally peer reviewed.

Open Access This is an Open Access article distributed in accordance with the Creative Commons Attribution Non Commercial (CC BY-NC 4.0) license, which permits others to distribute, remix, adapt, build upon this work non-commercially, and license their derivative works on different terms, provided the original work is properly cited and the use is non-commercial. See: http://creativecommons.org/ licenses/by-nc/4.0/

\section{REFERENCES}

1 Remzi FH, Church JM, Bast J, et al. Mucosectomy vs stapled ileal pouch-anal anastomosis in patients with familial adenomatous polyposis. Dis Col Rect 2001;44 (11):1590-6.

2 Culligan K, Walsh SR, Dunne C, et al. A histological and electron microscopic characterization of the mesenteric attachment of the colon prior to and after surgical mobilization. Ann Surg 2014;260:1048-56.

3 Culligan K, Remzi FH, Soop M, et al. Review of nomenclature in colonic surgeryproposal of a standardised nomenclature based on mesocolic anatomy. Surgeon 2013;2:1-5.

4 Culligan K, Coffey JC, Kiran RP, et al. The mesocolon: a prospective observational study. Colorect Dis 2012;14:421-30

5 Smith JC, Schäffer MW, Ballard BR, et al. Adenocarcinomas after prophylactic surgery for familial adenomatous polyposis. J Cancer Ther 2013;4:260-70.

6 Tajika M, Nakamura T, Nakahara O, et al. Risk of ileal pouch neoplasms in patients with familial adenomatous polyposis. World J Gastroenterol 2013;19:6774-83.

7 Ganshow P, Treiber I, Hinz U, et al. Residual rectal mucosa after stapled vs. handsewn ileal J-pouch-anal-anastomosis in patients with familial adenomatous polyposis (FAP) — a critical issue. Lagenbecks Arch Surg 2015;400:213-19.

8 Improved survival with preoperative radiotherapy in resectable rectal cancer: Swedish Rectal Cancer Trial. N Engl J Med 1997;336:980-7.

9 Kapiteijn E, Marijen CA, Nagtegaal ID, et al. Preoperative radiotherapy combined with total mesorectal excision for resectable rectal cancer. N Engl J Med 2001;345:638-46.

10 Sauer R, Becker $H$, Hohenberger W, et al. Preoperative versus postoperative chemoradiotherapy for rectal cancer. N Engl J Med 2004;351:1731-40.

11 Sloothaak DA, Geijsen DE, van Leersum NJ, et al., Dutch Surgical Colorectal Audit. Optimal time interval between neoadjuvant chemoradiotherapy and surgery for rectal cancer. Br J Surg 2013;100:933-9.

12 Bujko K, Nowacki MP, Nasierowska-Guttmejer A, et al. Long-term results of a radiation trial comparing preoperative short-course radiotherapy with preoperative conventionally fractionated chemoradiation for rectal cancer. Br J Surg 2006;93:1215-23.

13 Ngan SY, Burmeister B, Fisher RJ, et al. Randomised trial of short-course radiotherapy versus long-course chemoradiation comparing rates of local recurrence in patients with T3 rectal cancer: Trans-Tasman Radiation Oncology Group 01.04. J Clin Oncol 2012;30:3827-33. 
Copyright 2016 BMJ Publishing Group. All rights reserved. For permission to reuse any of this content visit http://group.bmj.com/group/rights-licensing/permissions.

BMJ Case Report Fellows may re-use this article for personal use and teaching without any further permission.

Become a Fellow of BMJ Case Reports today and you can:

- Submit as many cases as you like

- Enjoy fast sympathetic peer review and rapid publication of accepted articles

- Access all the published articles

- Re-use any of the published material for personal use and teaching without further permission

For information on Institutional Fellowships contact consortiasales@bmjgroup.com

Visit casereports.bmj.com for more articles like this and to become a Fellow 